\title{
Sex differences in risk factors for myocardial infarction: cohort study of UK Biobank participants
}

Check for updates

${ }^{1}$ The George Institute for Global Health, University of Oxford,

Oxford OX1 2BQ, UK

${ }^{2}$ Julius Center for Health

Sciences and Primary Care,

University Medical Center

Utrecht, Utrecht, Netherlands

${ }^{3}$ The George Institute for Global

Health, University of New South

Wales, Newtown, NSW, Australia

${ }^{4}$ Department of Epidemiology,

Johns Hopkins University,

Baltimore, MD, USA

Correspondence to: SA E Peters sanne.peters@georgeinstitute. ox.ac.uk

Additional material is published online only. To view please visit the journal online.

Cite this as: $B M J$ 2018;363:k4247 http://dx.doi.org/10.1136/bmj.k4247

Accepted: 25 September 2018

\author{
Elizabeth R C Millett, ${ }^{1}$ Sanne A E Peters, ${ }^{1,2}$ Mark Woodward ${ }^{1,3,4}$
}

\section{ABSTRACT}

OBJECTIVES

To investigate sex differences in risk factors for incident myocardial infarction (MI) and whether they vary with age.

DESIGN

Prospective population based study.

SETTING

UK Biobank.

\section{PARTICIPANTS}

471998 participants (56\% women; mean age 56.2 ) with no history of cardiovascular disease.

\section{MAIN OUTCOME MEASURE}

Incident (fatal and non-fatal) MI.

\section{RESULTS}

5081 participants (1463 (28.8\%) of whom were women) had MI over seven years' mean follow-up, resulting in an incidence per 10000 person years of 7.76 (95\% confidence interval 7.37 to 8.16 ) for women and 24.35 (23.57 to 25.16) for men. Higher blood pressure indices, smoking intensity, body mass index, and the presence of diabetes were associated with an increased risk of $\mathrm{Ml}$ in men and women, but associations were attenuated with age. In women, systolic blood pressure and hypertension, smoking status and intensity, and diabetes were associated with higher hazard ratios for $\mathrm{MI}$ compared with men: ratio of hazard ratios 1.09 (95\% confidence interval 1.02 to 1.16$)$ for systolic blood pressure, 1.55 (1.32 to 1.83 ) for current smoking, 2.91 (1.56 to 5.45 ) for type 1 diabetes, and 1.47 (1.16 to 1.87 ) for type 2 diabetes. There was no evidence that any of these ratios of hazard ratios decreased with age $(P>0.2)$. With the exception of type 1 diabetes, the incidence of MI was higher in men than in women for all risk factors.

\section{WHAT IS ALREADY KNOWN ON THIS TOPIC}

The incidence of myocardial infarction (MI) is lower in women than in men at younger ages, but the incidence becomes more similar with increasing age Meta-analyses have shown sex differences in the association between several risk factors and $\mathrm{Ml}$, but the studies included had varying levels of adjustment for confounders and could not examine sex differences by age group

\section{WHAT THIS STUDY ADDS}

Hypertension, smoking, and diabetes were associated with an increased risk of $\mathrm{MI}$ in women and men, but with an excess relative risk in women

Although the sex specific associations between these risk factors and $\mathrm{MI}$ attenuated with age, the excess relative risk of MI in women did not

Women and men should receive the same access to guideline based treatments for diabetes and hypertension, and to resources to help them lose weight and stop smoking

\section{CONCLUSIONS}

Although the incidence of MI was higher in men than in women, several risk factors were more strongly associated with $\mathrm{MI}$ in women compared with men. Sex specific associations between risk factors and MI declined with age, but, where it occurred, the higher relative risk in women remained. As the population ages and the prevalence of lifestyle associated risk factors increase, the incidence of $\mathrm{Ml}$ in women will likely become more similar to that in men.

\section{Introduction}

Coronary heart disease (CHD) has been the leading cause of mortality worldwide for over 25 years, ${ }^{12}$ and was estimated to be the cause of $17 \%$ of deaths globally in 2016. ${ }^{3}$ Death rates from CHD are considerably lower in women than in men at younger ages, but often converge with increasing age. Male-to-female coronary mortality rate ratios are typically around 4 to 5 in middle age (30-64) and 2 thereafter (65-89). ${ }^{4}$ In the INTERHEART case-control study, women had their first myocardial infarction (MI) on average nine years later than men. ${ }^{5}$ In addition to later presentation, men and women can have different symptoms, treatments, and outcomes of MI, some of which may be because of the effects and prevalence of risk factors. Several large scale meta-analyses have compared the sex specific associations between risk factors and CHD. Key findings from these analyses are that, compared with men, women had a higher ratio of relative risk of CHD: 44\% higher if they had diabetes ${ }^{6}$ and $25 \%$ higher if they were current smokers. ${ }^{7}$ However, these meta-analyses included studies performed over an extensive time scale, with heterogeneous study populations, and with varying sets of adjustment for potential confounders. Importantly, the analyses were unable to reliably explore whether the identified sex differences in relative risk were consistent with age, or to compare the sex differences for different risk factors on an equal basis. In addition, the analyses could not make comparisons on the absolute scale.

To obtain comparably adjusted results, overall and within age groups, we used the UK Biobank to investigate the sex differences in risk factors for MI and how these may vary with age.

\section{Methods}

The UK Biobank is a large prospective study of 502628 participants recruited between 2006 and $2010 .^{8}$ Participants aged between 40 and 69 were invited to attend one of 22 centres for a baseline assessment, where informed consent was obtained, a touchscreen questionnaire was completed, a face-to-face interview was conducted, and a range of physical measurements 
were taken. Participants gave details of their medical history, regular use of any drugs, and their lifestyle factors, such as smoking status. Baseline data are linked to hospital admissions data (hospital episode statistics admitted patient care activity (HES, England), the general/acute inpatient and day case dataset (SMR01, Scotland) and the patient episode database for Wales (PEDW)) and Office for National Statistics (ONS) mortality records, which enable long term follow-up of participants and their health outcomes.

Participants who subsequently withdrew from the study ( $n=64)$ and those with a history of cardiovascular disease (self reported or hospital admission diagnosis of MI, angina, or stroke before the date of the baseline assessment, $\mathrm{n}=30$ 566) were excluded from the current analyses.

\section{Measurement of risk factors}

We investigated six risk factors: blood pressure, smoking status, diabetes mellitus, body mass index, atrial fibrillation, and socioeconomic status. For blood pressure, the mean of two sitting systolic and diastolic blood pressure measurements, taken at baseline using the Omron HEM-7015IT digital blood pressure monitor (Omron Healthcare), was calculated. Blood pressure was categorised using American Heart Association (AHA) 2017 guidelines (normal: systolic blood pressure $<120 \mathrm{~mm} \mathrm{Hg}$ and diastolic blood pressure $<80$ mm Hg; elevated: systolic blood pressure 120-129 $\mathrm{mm} \mathrm{Hg}$ and diastolic blood pressure $<80 \mathrm{~mm} \mathrm{Hg}$; stage 1 hypertension: systolic blood pressure $130-139 \mathrm{~mm}$ $\mathrm{Hg}$ or diastolic blood pressure $80-89 \mathrm{~mm} \mathrm{Hg}$; and stage 2 hypertension: systolic blood pressure $\geq 140 \mathrm{~mm} \mathrm{Hg}$ or diastolic blood pressure $\geq 90 \mathrm{~mm} \mathrm{Hg}$ ). ${ }^{9}$ We further categorised AHA stages into eight groups according to reported use of antihypertensive drugs. Smoking status was self reported and included the daily cigarette consumption of current smokers. Self reported diabetes was categorised as type 1 if participants were aged less than 30 when the disease was diagnosed and they were using insulin, and type 2 otherwise. Participants with type 2 diabetes were further classified according to self reported treatment. Body mass index was calculated by dividing a participant's weight in kilograms, measured using the Tanita BC-418 MA body composition analyser (Tanita Corporation of America), by the square of his or her standing height in metres, measured using a Seca 202 height measure (SECA, Germany). Overweight was defined as a body mass index of at least $25 \mathrm{~kg} / \mathrm{m}^{2}$ and less than $30 \mathrm{~kg} / \mathrm{m}^{2}$; obesity was defined as a body mass index of $30 \mathrm{~kg} / \mathrm{m}^{2}$ or higher. A history of atrial fibrillation was self reported. Socioeconomic status was determined using the postcode based Townsend deprivation index and categorised into thirds using national cut-off points (high: $<-2.08$; middle $\geq-2.08$ to $<1.40$; low $\geq 1.40$ ).

\section{Outcomes}

The study endpoint was the incidence of fatal or nonfatal MI, defined using an algorithm developed by UK Biobank. ${ }^{10}$ Linking with relevant hospital admissions data (HES, SMR01, and PEDW) and ONS enabled UK Biobank to identify the date of the first known MI after the date of baseline assessment by using codes I21, I22, I23, I24.1 or I25.2 from the 10th edition of the International Classification of Diseases. Follow-up for all participants started at inclusion in the UK Biobank and ended on 31 March 2016, or when the first fatal or non-fatal MI occurred.

\section{Statistical analyses}

Baseline characteristics for women and men are presented as number (percentage) for categorical variables, mean (standard deviation) for approximately symmetrical continuous variables, and median (interquartile range) for asymmetrical continuous variables. The incidence of MI was estimated separately for women and men. Cox proportional hazard models were used to estimate hazard ratios and 95\% confidence intervals for MI comparing women with men, adjusted for age, systolic blood pressure, body mass index, smoking status, and diabetes. These analyses were further stratified by age in five year groups.

To estimate the hazard ratios for MI for each risk factor, we used Cox regression models, with interaction terms between each variable and sex. All models were adjusted for age and other variables specified a priori, which varied across risk factors. In addition to age, we adjusted systolic blood pressure, diabetes, and socioeconomic status for each other as well as for smoking status, body mass index, use of lipid lowering drugs, and antihypertensive drugs. Atrial fibrillation was similarly adjusted for these eight variables. Diastolic blood pressure and AHA hypertension stages were adjusted for the same variables as systolic blood pressure, except when AHA hypertension stage was further categorised by use of antihypertensive drugs, in which case adjustment for antihypertensive drugs was clearly inappropriate. The models for smoking variables included socioeconomic status, and the models for body mass index included smoking status and socioeconomic status. We decided not to adjust body mass index for systolic blood pressure because systolic blood pressure is thought to be a mediating factor and our goal was to examine the independent effects of sex on risk factor associations with MI.

The interaction term of each risk factor with sex was used to obtain the women-to-men relative hazard ratio for each risk factor. This ratio should be interpreted as a measure of interaction (in a statistical sense) or effect modification (in a clinical sense). We used a likelihood ratio test to check for deviation from the linear trend for the ratio of hazard ratios for categories of daily cigarette consumption.

Missing data can be a source of bias. We determined the percentage of missing data for each variable used in this study. We ran a sensitivity analysis for any variable for which more than $5 \%$ of the data were missing by performing 20 iterations of a multiple imputation using chained equations and including sex interactions in the imputation model. ${ }^{11}$ When less than 
$5 \%$ of data for a risk factor or potential confounder of interest was missing, participants with missing data were not included in the relevant model.

To investigate whether sex differences in risk factors differed by age group $(<50,50-59$, and $\geq 60)$, we added a three way interaction between sex, age group, and the risk factor of interest to the models. Age group was subsequently included as a linear term to produce Wald test $\mathrm{P}$ values for trend among women and men and in the interaction for the ratio of hazard ratios. Because the UK Biobank cohort is comparatively socially advantaged (and healthy) compared with the UK population in general, we investigated whether there was evidence of heterogeneity in the effects of risk factors according to socioeconomic status. Therefore, we ran additional subgroup analyses for participants at or above, and below the national median Townsend deprivation index score $(-0.56)$.

We also looked at sex differences on the absolute scale, which are less likely to be relevant than relative risks for application in other populations, but should be considered when making clinical decisions. We evaluated sex differences on the absolute scale as unadjusted and adjusted rates per 10000 person years by sex and their women-to-men difference of differences, estimated using Poisson regression models. Adjustments were the same as those used in the Cox models.

Analyses were performed using Stata version 14.2.

\section{Patient and public involvement}

No patients were involved in setting the research question or the outcome measures, and they were not involved in developing plans for the design or implementation of the study. No patients were asked to advise on interpretation or writing up of results. We have no plans to disseminate the results of the research to study participants or the relevant patient community.

\section{Results}

Table 1 presents the baseline characteristics of 471998 participants with no history of cardiovascular disease included in these analyses. The proportion of women was $56 \%$ and the mean age at study baseline was 56 (standard deviation 8) in both sexes. At baseline, a lower percentage of women than men had diabetes or atrial fibrillation, or were taking lipid lowering or blood pressure lowering drugs. Overall, women had slightly lower blood pressure and were less likely to have ever smoked than men.

After a mean follow-up of seven years, 5081 first MI events were recorded (1463 (28.8\%) in women) (table 1). Models adjusted for multiple variables showed that the rate of MI in women was less than half that in men (hazard ratio $0.37 ; 95 \%$ confidence interval 0.35 to 0.40 ). The lower risk of MI in women compared with men was apparent across all age groups, but attenuated slightly with increasing age, from 0.27 (0.18 to 0.41$)$ among those aged less than
45, to 0.45 (0.40 to 0.50$)$ among those aged 65 and over (supplementary table 1 ).

\section{Blood pressure}

Rising values of all blood pressure indices were associated with a higher risk of MI in both sexes after adjusting for confounding variables (fig 1). Compared with normal blood pressure, the risk of MI increased with AHA hypertension stage in both sexes and was consistently higher among women than men. The women-to-men ratio of hazard ratios for elevated blood pressure was 1.83 (95\% confidence interval 1.33 to 2.52), whereas for stage 1 and stage 2 hypertension the ratios were both about 1.5 (fig 2). We also considered the effects of higher blood pressure on MI according to antihypertensive drug use. The hazard ratios for MI were higher for participants taking drugs than for those not taking drugs for each AHA stage and in both sexes (supplementary table 2). This increase was more pronounced in women. For example, comparing participants with elevated blood pressure taking antihypertensive drugs with those with normal blood pressure not taking antihypertensive drugs, the hazard ratio was 3.65 (2.44 to 5.44$)$ in women and 1.75 (1.26 to 2.44 ) in men (ratio of hazard ratios $2.08,95 \%$ confidence interval 1.24 to 3.50 ) (supplementary table 2).

\section{Smoking status}

Compared with never smoking, current and former smoking were each associated with an increased risk of MI in both sexes, but with larger hazard ratios in women than men. For current smokers, the hazard ratios were 3.46 (95\% confidence interval 3.02 to 3.98) in women and 2.23 (2.03 to 2.44) in men (fig 1) (ratio of hazard ratios 1.55 , 95\% confidence interval 1.32 to 1.83 ; fig 2). Greater smoking intensity was associated with a higher risk of MI in both sexes, but especially among women. The women to men ratio of hazard ratios increased with cigarette consumption (with evidence of non-linearity, $\mathrm{P}=0.006$; fig 2).

\section{Type 1 and 2 diabetes}

Compared with people without diabetes, women with type 1 diabetes had more than eight times the risk of MI (hazard ratio $8.18,95 \%$ confidence interval 5.20 to 12.86; fig 1), almost three times the hazard ratio in men (2.81, 1.82 to 4.33; fig 1 ; ratio of hazard ratios 2.91, 1.56 to 5.45 ; fig 2). Type 2 diabetes was associated with an increased risk of MI in both sexes; the hazard ratio was 1.96 (1.60 to 2.41) in women and 1.33 (1.18 to 1.51 ) in men (fig 1); the ratio of hazard ratios was 1.47 (1.16 to 1.87 ; fig 2 ). When analysing the type of treatment given to people with type 2 diabetes, there was still an excess risk in women compared with men (supplementary table 3). Hazard ratios for both sexes and the women-to-men ratio were greater when insulin use was reported.

\section{Body mass index}

The risk of MI rose with increasing body mass index, and with being overweight or obese (compared with 


\begin{tabular}{|c|c|c|}
\hline Characteristics & Women $(n=263323)$ & Men $(n=208675)$ \\
\hline Incident myocardial infarction during follow-up, No (\% of total MIs) & $1463(28.8)$ & $3618(71.2)$ \\
\hline Mean (SD) age (years) & $56.2(8.0)$ & $56.3(8.2)$ \\
\hline \multicolumn{3}{|l|}{ Ethnicity: } \\
\hline White & $248045(94.2)$ & $195990(93.9)$ \\
\hline Other* & $14050(5.3)$ & $11302(5.4)$ \\
\hline \multicolumn{3}{|l|}{ Blood pressure $(\mathrm{mm} \mathrm{Hg})$ : } \\
\hline Mean (SD) systolic & $135.2(19.2)$ & $141.1(17.4)$ \\
\hline Mean (SD) diastolic & $80.8(10.0)$ & $84.5(9.9)$ \\
\hline \multicolumn{3}{|l|}{ AHA hypertension categories: } \\
\hline Normal & $53770(20.5)$ & $17316(8.3)$ \\
\hline Elevated & $34192(13.0)$ & $23256(11.2)$ \\
\hline Stage 1 hypertension & $115930(44.1)$ & $103317(49.6)$ \\
\hline Stage 2 hypertension & $58707(22.4)$ & $64278(30.9)$ \\
\hline \multicolumn{3}{|l|}{ Smoking status: } \\
\hline Never smoker & $157200(60.0)$ & $104720(50.5)$ \\
\hline Former smoker & $81611(31.2)$ & $76922(37.1)$ \\
\hline Current smoker & $23083(8.8)$ & $25776(12.4)$ \\
\hline Mean (SD) cigarettes smoked daily (current smokers) & $14(7.0)$ & $17(9.0)$ \\
\hline \multicolumn{3}{|l|}{ Smoking intensity (average No of cigarettes smoked daily): } \\
\hline $1-9$ & $4241(18.4)$ & $2571(10.0)$ \\
\hline $10-19$ & $7953(34.5)$ & $6262(24.3)$ \\
\hline$\geq 20$ & $5200(22.5)$ & $6961(27.0)$ \\
\hline Not reported & $5689(24.6)$ & $9982(38.7)$ \\
\hline Mean (SD) years smoked (current smokers) & $36.0(9.3)$ & $36.9(9.9)$ \\
\hline \multicolumn{3}{|l|}{ Body mass index $\left(\mathrm{kg} / \mathrm{m}^{2}\right)$ : } \\
\hline Mean (SD) body mass index & $27.0(5.2)$ & $27.7(4.2)$ \\
\hline Overweight $(\geq 25,<30)$ & $96136(36.7)$ & $102942(49.7)$ \\
\hline Obese $(\geq 30)$ & $60343(23.0)$ & $50364(24.3)$ \\
\hline \multicolumn{3}{|l|}{ Socioeconomic status: } \\
\hline Median (interquartile range) Townsend deprivation index score & $-2.17(-3.65-0.42)$ & $-2.17(-3.67-0.54)$ \\
\hline \multicolumn{3}{|l|}{ Townsend deprivation thirds: } \\
\hline Low & $49396(18.8)$ & $41000(19.7)$ \\
\hline Middle & $78929(30.0)$ & $60777(29.2)$ \\
\hline High & $134681(51.2)$ & $106625(51.2)$ \\
\hline \multicolumn{3}{|l|}{ Diabetes: } \\
\hline Type $1 \dagger$ & $500(0.2)$ & $531(0.3)$ \\
\hline Type 2 & $8631(3.3)$ & $11852(5.7)$ \\
\hline \multicolumn{3}{|l|}{ Type 2 treatment: } \\
\hline No drugs reported & $3497(40.5)$ & $4275(36.1)$ \\
\hline Oral drugs only & $4000(46.3)$ & $6018(50.8)$ \\
\hline Insulin only & $492(5.7)$ & $670(5.7)$ \\
\hline Oral drugs and insulin & $642(7.4)$ & $889(7.5)$ \\
\hline Mean (SD) type 2 diagnosis (years) & $6.7(7.2)$ & $7.2(8.1)$ \\
\hline \multicolumn{3}{|l|}{ Atrial fibrillation: } \\
\hline History of atrial fibrillation & $985(0.4)$ & $2006(1.0)$ \\
\hline \multicolumn{3}{|l|}{ Drug use: } \\
\hline Antihypertensive drugs & $32340(12.3)$ & $32563(15.6)$ \\
\hline Lipid lowering drugs & $23067(8.8)$ & $29544(14.2)$ \\
\hline \multicolumn{3}{|c|}{$\begin{array}{l}\text { AHA=American Heart Association. } \\
\text { *Includes Asian or Asian British, black or black British, Caribbean, African, any other black background, Chinese, other ethnic group, white } \\
\text { and black Caribbean, white and black African, white and Asian, any other mixed background, Indian, Pakistani, Bangladeshi, any other Asian } \\
\text { background. } \\
\text { tDefined as diagnosis at age }<30 \text { and receiving insulin treatment. }\end{array}$} \\
\hline
\end{tabular}

normal weight) for both sexes (fig 1). However, there was no evidence of any difference by sex (ratio of hazard ratios 0.97 (95\% confidence interval 0.91 to 1.03) for every $5 \mathrm{~kg} / \mathrm{m}^{2}$ additional body mass index, 0.89 ( 0.76 to 1.03 ) for overweight, and 0.93 ( 0.79 to 1.09) for obese; fig 2).

\section{Atrial fibrillation}

The hazard ratio for MI associated with atrial fibrillation was 1.54 (95\% confidence interval 0.89 to 2.66 ) in women and 1.35 (1.04 to 1.77 ) in men (fig 1); there was no evidence of a sex difference (ratio of hazard ratios $1.14,95 \%$ confidence interval 0.62 to 2.09; fig 2).

\section{Socioeconomic status}

Compared with high socioeconomic status, women with a low socioeconomic status had a hazard ratio for MI of 1.27 (95\% confidence interval 1.11 to 1.46 ); the equivalent hazard ratio in men was 1.12 (1.03 to 1.22) (fig 1). For each unit increase in the Townsend deprivation index score (moving from higher to lower 


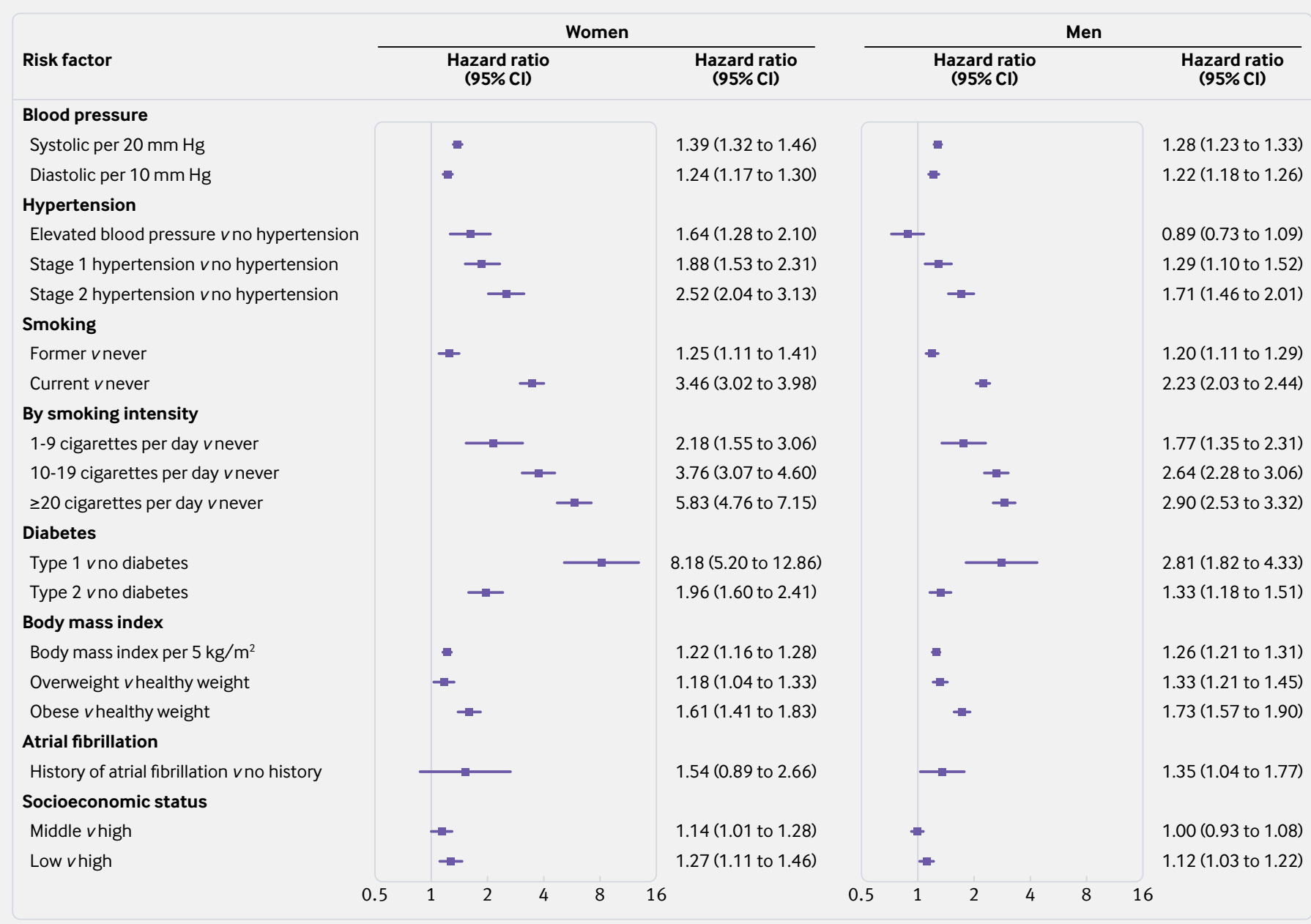

Fig 1 | Adjusted hazard ratios for association between risk factors and incident myocardial infarction by sex. Horizontal lines indicate corresponding $95 \%$ confidence intervals around hazard ratios. All models were adjusted for age. Additionally, systolic blood pressure, diabetes, and socioeconomic status were adjusted for each other as well as smoking status, body mass index, lipid lowering drugs, and antihypertensive drugs. Atrial fibrillation was similarly adjusted for these eight variables. Diastolic blood pressure and American Heart Association hypertension stages were adjusted for the same variables as systolic blood pressure. Models for smoking variables included socioeconomic status, and models for body mass index contained smoking status and socioeconomic status

socioeconomic status), women had 3\% (hazard ratio $1.03,1.02$ to 1.05$)$ higher risk of MI compared with a $2 \%(1.02,1.01$ to 1.03$)$ higher risk in men. There was some evidence that this risk was slightly higher among women than men (ratio of hazard ratios 1.02, 1.00 to 1.04).

\section{Confounding and modification by age}

The results from the age adjusted analyses were generally similar to the multiple adjusted analyses (supplementary table 4). The sex specific hazard ratios for stage 1 and 2 hypertension, current smokers, diabetes, and socioeconomic status were slightly higher than those that were further adjusted, as were the ratios of hazard ratios for current smokers, diabetes, atrial fibrillation, and the lowest third category of socioeconomic status.

In both sexes, the hazard ratios for systolic blood pressure, diastolic blood pressure, hypertension stage 2, and high smoking intensity decreased with increasing age (fig 3). Type 2 diabetes was associated with a higher risk of MI in those aged less than 50 (women: hazard ratio 3.73 (95\% confidence interval 1.93 to 7.20 ); men: 2.18 (1.43 to 3.31); fig 3), while the 50-59 and 60 and older age groups had similarly increased risks of around 90\% in women and 30\% in men. For none of the risk factors investigated was there evidence that the women-to-men ratio of hazard ratios differed by age group (fig 4 and supplementary table 5).

\section{Modification by socioeconomic status}

The only evidence of heterogeneity by socioeconomic status in the effects of sex on risk factors for MI was for former smoking versus never smoking $(\mathrm{P}=0.004$, supplementary table 6). Among women with low socioeconomic status, former smokers had a $49 \%$ increased risk of MI compared with non-smokers. This increase was not present among men (hazard ratio 1.06, $95 \%$ confidence interval 0.93 to 1.22 ; ratio of 


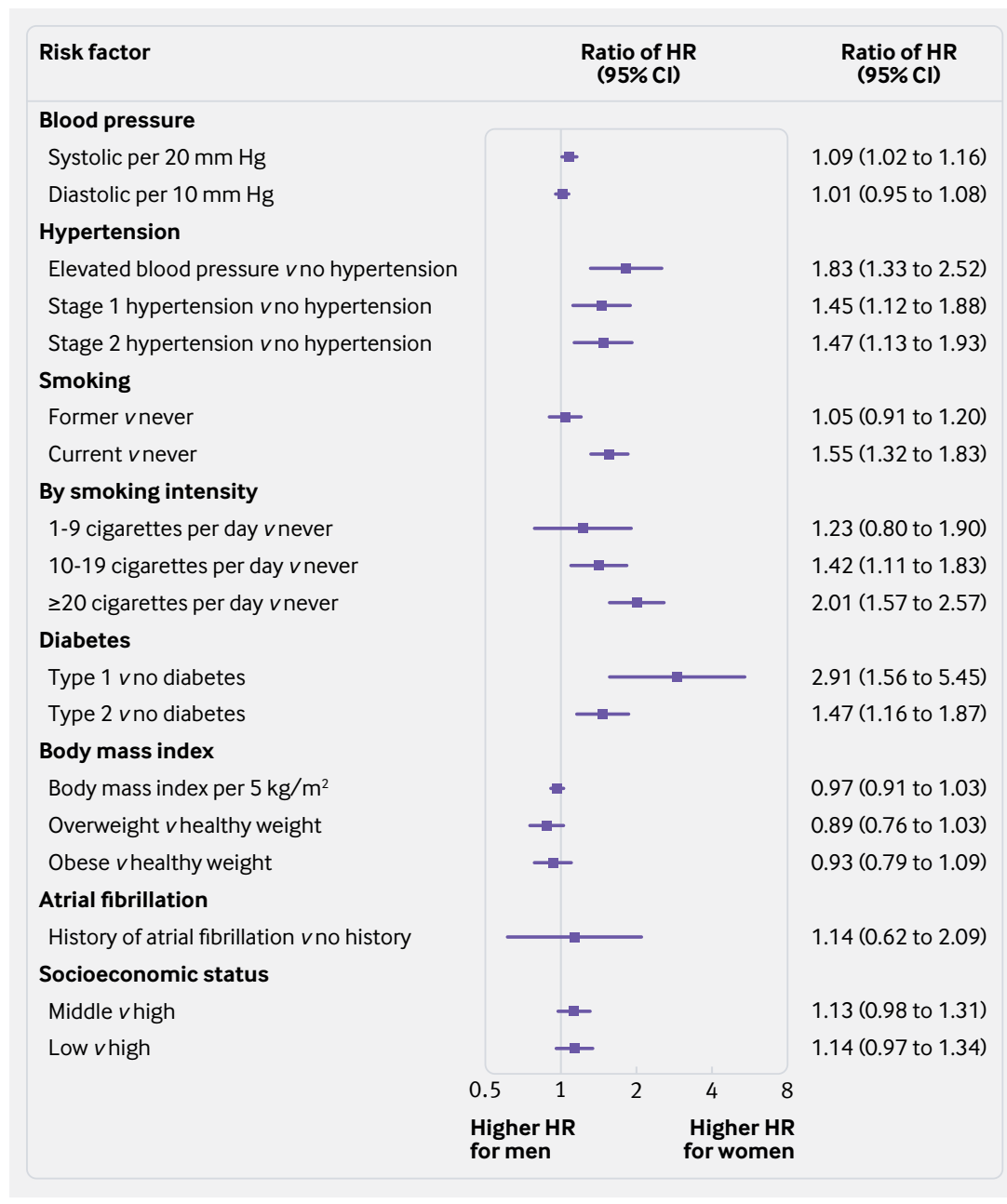

Fig 2 | Adjusted women-to-men ratios of hazard ratios for association between risk factors and incident myocardial infarction. Horizontal lines indicate corresponding $95 \%$ confidence intervals around ratio of hazard ratios. All models were adjusted for age. Additionally, systolic blood pressure, diabetes, and socioeconomic status were adjusted for each other as well as smoking status, body mass index, lipid lowering drugs, and antihypertensive drugs. Atrial fibrillation was similarly adjusted for these eight variables. Diastolic blood pressure and American Heart Association hypertension stages were adjusted for the same variables as systolic blood pressure. Models for smoking variables included socioeconomic status, and models for body mass index contained smoking status and socioeconomic status

hazard ratios $1.41,1.11$ to 1.79$)$. There was no evidence of a sex difference in the hazard ratios comparing former with never smokers among those with high socioeconomic status (supplementary table 6).

\section{Sex comparisons of rates of $\mathrm{MI}$}

Finally, we describe sex differences on the absolute scale. Incidence rates of MI per 10000 person years were 7.76 (95\% confidence interval 7.37 to 8.16 ) in women and 24.35 (23.57 to 25.16) in men. Type 1 diabetes was the only risk factor associated with comparable rates of MI in women and men. For all other risk factors, and for every category of these risk factors, men had higher rates of MI than women (table 2; supplementary table 7). The unadjusted rates of MI were generally similar to the multiple adjusted rates, except among participants with type 1 diabetes in whom rates increased after adjustment, and among those with type 2 diabetes or atrial fibrillation in whom rates decreased after adjustment. In both sexes, multiple adjusted rates were highest among those with type 1 diabetes (women: 64.92/10000 person years (95\% confidence interval 35.88 to 93.96$)$; men: $61.92 / 10000$ person years (35.17 to 88.67 ); table 2 ).

\section{Discussion}

This study of 471998 middle aged UK Biobank participants with no history of cardiovascular disease analysed the sex differences in risk factors for myocardial infarction (MI). Women who smoked more than 20 cigarettes per day had twice the relative risk of MI than equivalent men, and elevated blood pressure was associated with a more than $80 \%$ higher relative risk in women. Hypertension stages 1 and 2, smoking 10-19 cigarettes daily, and type 2 diabetes each were $40 \%$ more strongly associated with the risk of MI in women than men.

Since the sex specific relative risks attenuated with age in both sexes, our results suggest that cardiovascular risk scores should consider including age interactions for greater predictive accuracy. However, we found no evidence to suggest that the women-to-men comparisons were significantly different across age groups.

\section{Strengths and limitations of this study}

Previous studies of sex differences in MI have been restricted to a single risk factor, pooled data from disparate studies, or were based on hospital populations. In this study we analysed sex differences in MI across a range of risk factors in a general population using standardised methods on the relative and absolute scales. We also examined how age impacts sex differences in MI. The only comparable study examining a similar range of risk factors in men and women and using a standard protocol is INTERHEART. This, however, was a retrospective case-control study of patients admitted to hospital with their first acute MI, and hospital sourced controls. The study design is inferior to the UK Biobank cohort design and is more susceptible to bias. ${ }^{12}$

Our study has limitations. Some variables had missing data but the only variable with more than $5 \%$ of data missing was the number of cigarettes smoked daily (supplementary table 8). After multiple imputation, the effects of smoking intensity, however, were not appreciably different from the complete case analysis (supplementary table 9) and we conclude that the primary results are valid. Most UK Biobank participants are white and further work is required to assess the generalisability of the results to other populations. Higher socioeconomic status was over represented in the study population, which could have limited the ability of this study to find sex differences among different socioeconomic groups. Blood samples were taken from all participants but at the time of analysis the lipid profile data were not available, 


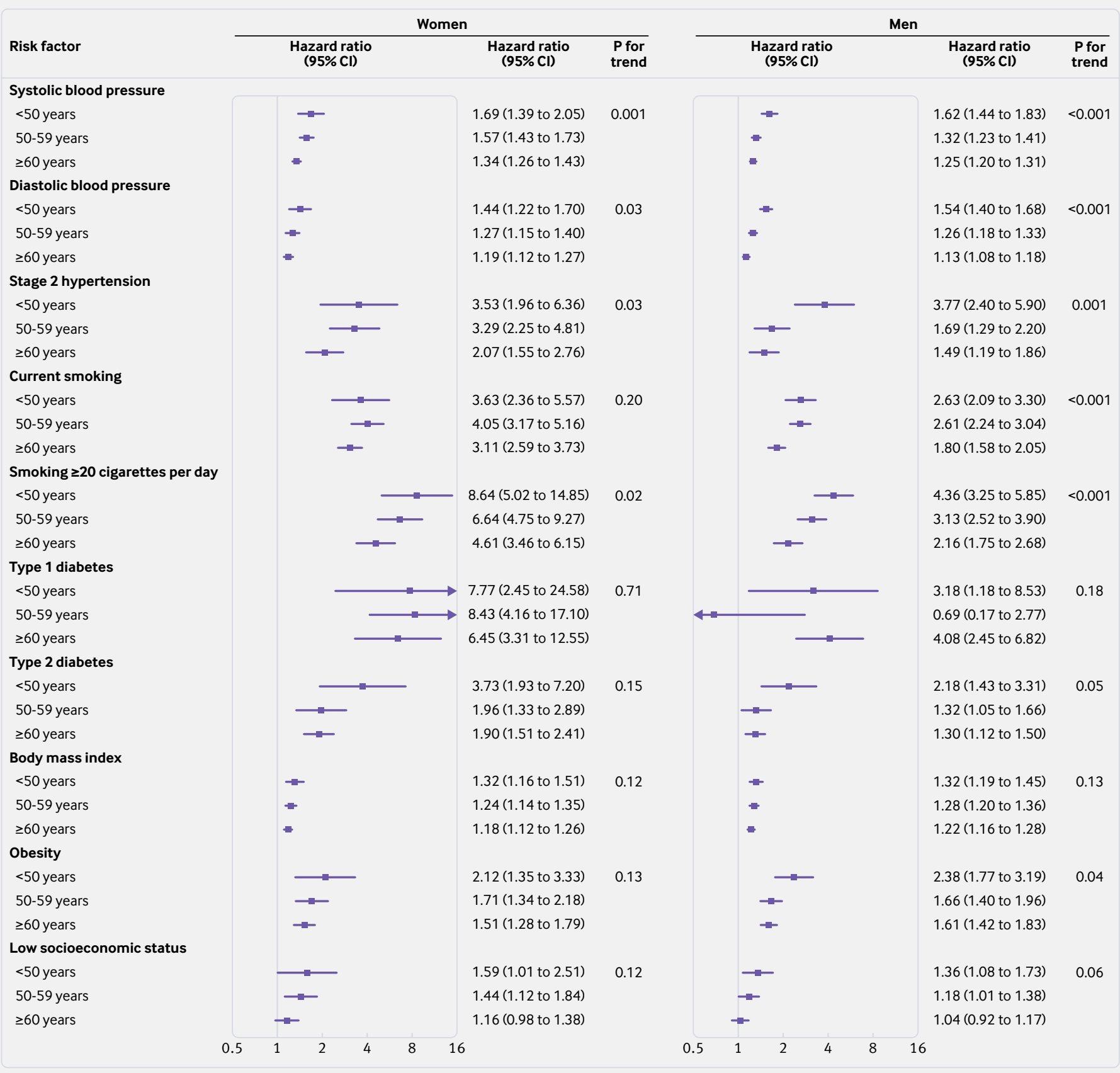

Fig 3 | Adjusted hazard ratios for association between risk factors and incident myocardial infarction by age group and sex. Horizontal lines indicate corresponding $95 \%$ confidence intervals around hazard ratios. Systolic blood pressure is given per $20 \mathrm{~mm} \mathrm{Hg}$ and diastolic blood pressure per 10 $\mathrm{mm}$ Hg. Participants with stage 2 hypertension were compared with participants with normal blood pressure; current smokers were compared with never smokers; participants with diabetes were compared with those without diabetes; obesity was compared with body mass index less than $25 \mathrm{~kg} / \mathrm{m}^{2}$; and for socioeconomic status the lowest third was compared with the highest third. Models for systolic blood pressure, diabetes and socioeconomic status were adjusted for each other as well as smoking status, body mass index, lipid lowering drugs, and antihypertensive drugs. Diastolic blood pressure and American Heart Association hypertension stages were adjusted for the same variables as systolic blood pressure. Models for smoking variables included socioeconomic status, and models for body mass index contained smoking status and socioeconomic status

preventing examination of this as a risk factor and adjusting for it as a confounder. Instead we adjusted analyses for participants' use of lipid lowering drugs when appropriate, but residual confounding by lipid levels is possible. Diagnoses and recording of drug use at baseline relied on self report, which will have resulted in some errors. However, these errors were probably minor and may have been the same in both sexes. UK Biobank participants were aged between 40 and 70 at recruitment, and so sex differences among younger and older populations were not analysed. Whether the additional risk associated with some factors varies across a wider age range would be an interesting topic for future research. 


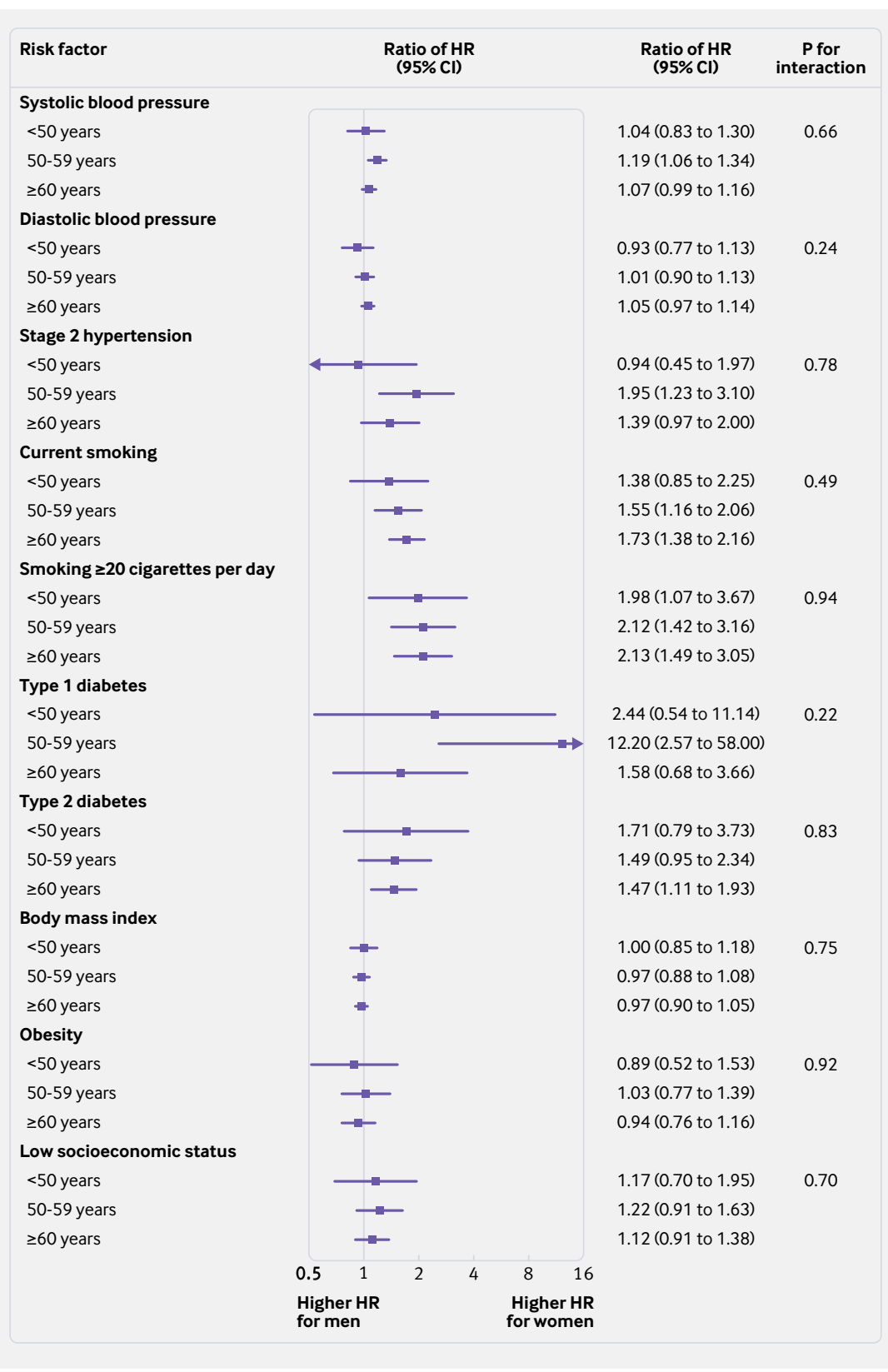

Fig 4 | Adjusted women-to-men ratios of hazard ratios for association between risk factors and incident myocardial infarction by age group. Horizontal lines indicate corresponding $95 \%$ confidence intervals around ratio of hazard ratios. Systolic blood pressure is given per $20 \mathrm{~mm} \mathrm{Hg}$ and diastolic blood pressure per $10 \mathrm{~mm} \mathrm{Hg}$. Participants with stage 2 hypertension were compared with participants with normal blood pressure; current smokers were compared with never smokers; participants with diabetes were compared with those without diabetes; obesity was compared with body mass index less than $25 \mathrm{~kg} / \mathrm{m}^{2}$; and for socioeconomic status the lowest third was compared with the highest third. Models for systolic blood pressure, diabetes, and socioeconomic status were adjusted for each other as well as smoking status, body mass index, lipid lowering drugs, and antihypertensive drugs. Diastolic blood pressure and American Heart Association hypertension stages were adjusted for the same variables as systolic blood pressure. Models for smoking variables included socioeconomic status, and models for body mass index contained smoking status and socioeconomic status people, the increased risk of ischaemic heart disease with each $10 \mathrm{~mm} \mathrm{Hg}$ rise in systolic blood pressure was found to be $13 \%$ in women and men. ${ }^{13}$ There was important heterogeneity between studies, which could partly be because of different trends in blood pressure worldwide. ${ }^{14}$ Our findings are similar to those from a recent English study of 1.25 million patients and 11029 MI events, in which a slightly higher relative risk of MI with increasing systolic blood pressure, but not diastolic blood pressure, was found in women compared with men. ${ }^{15}$ INTERHEART reported higher odds ratios of MI in women with hypertension than in men with hypertension (odds ratio 2.95 (95\% confidence interval 2.66 to 3.28 ) in women and 2.32 (2.16 to 2.48 ) in men). ${ }^{5}$ We found a higher relative risk of MI in women compared with men across all American Heart Association (AHA) hypertension stages, and this was most pronounced among those with elevated blood pressure. Women may be less likely to receive blood pressure lowering drugs and be less compliant with treatment, resulting in poorer blood pressure control than men. ${ }^{16}$ Women's longer exposure to the effects of hypertension (including before any treatment) could explain some of the higher relative risk we found in our analyses.

The INTERHEART study found that smoking accounted for around $36 \%$ of the population attributable risk of MI worldwide. ${ }^{12}$ Both men and women who were current smokers had around three times the odds of MI compared with never smokers. There was evidence of a sex difference by former smoking status. Women who were former smokers were not found to have increased odds of MI compared with never smokers, whereas men who were former smokers had around $60 \%$ increased odds of MI compared with never smokers. ${ }^{5}$ Our previous metaanalysis described a women to men ratio of relative risks of CHD of 1.25 (95\% confidence interval 1.12 to 1.39) in current smokers compared with nonsmokers, ${ }^{7}$ which is lower than the ratio of hazard ratios of 1.55 in current smokers compared with never smokers found in the current study. In agreement with the current study, this meta-analysis found no evidence of a sex difference when comparing the risk of CHD between former smokers and never smokers. ${ }^{7}$ INTERHEART and the Troms $\emptyset$ study reported an association between rising cigarette consumption and an increasing risk of MI in both sexes. ${ }^{17}{ }^{18}$ Although the Troms $\emptyset$ study reported no evidence of an interaction between sex and smoking intensity, the effect sizes in both studies were larger among women than men. ${ }^{17} 18$ Our estimate of a higher relative risk in female, than male, current smokers compared with never smokers, and with increasing cigarette consumption, may be explained by differences in the duration of the tobacco epidemic worldwide; in many countries the epidemic among women is relatively recent, leading to an underestimate of women's excess relative risk in some studies. ${ }^{1920}$ We have previously described how the smoking habits in UK Biobank men and women have become increasingly similar over time, ${ }^{21}$ providing a
Comparison with other studies

This study adds to the growing literature on potential sex differences in risk factors for cardiovascular disease. In a meta-analysis of more than 900000 


\begin{tabular}{|c|c|c|c|}
\hline \multirow[b]{2}{*}{ Variables } & \multicolumn{2}{|c|}{ Rates/10 000 person years $(95 \% \mathrm{Cl})$} & \multirow{2}{*}{$\begin{array}{l}\text { Difference of rate } \\
\text { differences }(95 \% \mathrm{Cl})\end{array}$} \\
\hline & Women $(n=263323)$ & Men $(n=208675)$ & \\
\hline \multicolumn{4}{|l|}{ AHA hypertension categories: } \\
\hline Normal & $4.32(3.49$ to 5.15$)$ & 16.88 (14.33 to 19.43$)$ & - \\
\hline Elevated & $7.07(5.92$ to 8.21$)$ & 15.04 (13.05 to 17.03$)$ & 4.59 (1.09 to 8.09$)$ \\
\hline Stage 1 hypertension & $8.23(7.60$ to 8.87$)$ & 21.85 (20.79 to 22.91$)$ & $-1.05(-4.00$ to 1.90$)$ \\
\hline Stage 2 hypertension & 11.18 (10.19 to 12.18$)$ & $28.92(27.42$ to 30.41$)$ & $-5.17(-8.42$ to -1.92$)$ \\
\hline \multicolumn{4}{|l|}{ Smoking status: } \\
\hline Never smoker & $6.09(5.62$ to 6.55$)$ & 19.84 (18.79 to 20.90$)$ & - \\
\hline Former smoker & $7.63(6.94$ to 8.31$)$ & $23.76(22.52$ to 24.99$)$ & $-2.37(-4.20$ to -0.55$)$ \\
\hline Current smoker & 21.01 (18.61 to 23.41$)$ & $44.06(40.83$ to 47.29$)$ & $-9.29(-13.49$ to -5.10$)$ \\
\hline \multicolumn{4}{|c|}{$\begin{array}{l}\text { Smoking intensity (average No of cigarettes } \\
\text { smoked daily): }\end{array}$} \\
\hline Never $^{\star}$ & $5.70(5.26$ to 6.13$)$ & 18.99 (17.98 to 19.99 ) & - \\
\hline $1-9$ & $12.41(8.29$ to 16.53$)$ & 33.62 (24.79 to 42.46$)$ & $-7.93(-17.74$ to 1.89$)$ \\
\hline 10-19 & 21.33 (17.37 to 25.28$)$ & 49.95 (43.11 to 56.79$)$ & $-15.34(-23.33$ to -7.35$)$ \\
\hline$\geq 20$ & $33.00(26.82$ to 39.17$)$ & $54.73(47.86$ to 61.59$)$ & $-8.44(-17.76$ to 0.88$)$ \\
\hline \multicolumn{4}{|l|}{ Diabetes: } \\
\hline No diabetes & 7.95 (7.50 to 8.40$)$ & $22.06(21.27$ to 22.84$)$ & - \\
\hline Type $1^{\star}$ & $64.92(35.88$ to 93.96$)$ & $61.92(35.17$ to 88.67$)$ & $17.11(-22.39$ to 56.61$)$ \\
\hline Type 2 & $15.63(12.64$ to 18.62$)$ & $29.41(25.92$ to 32.91$)$ & $0.33(-4.40$ to 5.05$)$ \\
\hline \multicolumn{4}{|l|}{ Body mass index $\left(\mathrm{kg} / \mathrm{m}^{2}\right)$ : } \\
\hline Normal $(<25)$ & $6.66(6.04$ to 7.28$)$ & 17.62 (16.30 to 18.93$)$ & - \\
\hline Overweight $(\geq 25,<30)$ & 7.82 (7.16 to 8.49$)$ & 23.38 (22.28 to 24.48$)$ & $-4.59(-6.53$ to -2.65$)$ \\
\hline Obesity $(\geq 30)$ & 10.69 (9.69 to 11.68$)$ & 30.47 (28.66 to 32.29 ) & $-8.83(-11.36$ to -6.30$)$ \\
\hline \multicolumn{4}{|l|}{ Atrial fibrillation: } \\
\hline None & $8.59(8.13$ to 9.04$)$ & 22.47 (21.71 to 23.23$)$ & - \\
\hline History of atrial fibrillation & $13.19(5.97$ to 20.40$)$ & 30.41 (22.37 to 38.45$)$ & $-3.34(-14.18$ to 7.50$)$ \\
\hline \multicolumn{4}{|l|}{ Townsend deprivation thirds: } \\
\hline High & $7.84(7.21$ to 8.46$)$ & $22.00(20.97$ to 23.03$)$ & - \\
\hline Middle & 8.93 (8.10 to 9.77$)$ & 22.11 (20.73 to 23.50$)$ & $0.98(-1.03$ to 2.99$)$ \\
\hline Low & 10.00 (8.92 to 11.08$)$ & 24.66 (22.86 to 26.45$)$ & $-0.50(-2.92$ to 1.93$)$ \\
\hline \multicolumn{4}{|c|}{$\begin{array}{l}\text { AHA=American Heart Association. } \\
\text { All models were adjusted for age. Additionally, systolic blood pressure, diabetes, and socioeconomic status were adjusted for each other as well as } \\
\text { smoking status, body mass index, lipid lowering drugs, and antihypertensive drugs. Atrial fibrillation was similarly adjusted for these eight variables. } \\
\text { Diastolic blood pressure and AHA hypertension stages were adjusted for the same variables as systolic blood pressure. The models for smoking variables } \\
\text { included socioeconomic status, and the models for body mass index contained smoking status and socioeconomic status. } \\
\text { *Smoking status and smoking intensity models produced slightly different adjusted rates of MI for never smokers. This is because of the effect of } \\
\text { adjustment for covariables differing slightly in each model, as they contained different smoking variables, and former smokers and those with missing } \\
\text { cigarette consumption were excluded from the smoking intensity model. }\end{array}$} \\
\hline
\end{tabular}

more direct women-to-men comparison of the risk of MI associated with smoking.

The sex difference in risk of cardiovascular disease in people with diabetes has been subject to increasing interest over recent years. Pooled analyses of over 800000 people, including over 26000 incident CHD events, showed that women with diabetes had a $44 \%$ excess relative risk of incident CHD compared with men with diabetes. ${ }^{6}$ INTERHEART and an Italian cohort study reported higher risks of MI in women with diabetes than in men, ${ }^{522}$ whereas a meta-analysis and the China Kadoorie Biobank described an excess relative risk of mortality from $\mathrm{CHD}$ in women with diabetes compared with men. ${ }^{23} 24$ In contrast, a large English cohort study of 1.9 million patients observed a slightly increased risk of non-fatal MI in women with type 2 diabetes aged less than 60 than in men of a similar age, but no differences among older age groups. ${ }^{25}$ Exclusion of fatal MI may be partly responsible for their results, as MI mortality has been found to be higher in women than in men with diabetes. ${ }^{26}$ Deterioration in cardiovascular risk factor levels among those with and without type 2 diabetes is greater in women than in men; therefore women with diabetes are at a disadvantage compared with men, even before their diagnosis. ${ }^{27}$ Additionally, in the UK women with diabetes are $15 \%$ less likely than men with diabetes to meet all recommended care requirements, and might be less likely to achieve target values for treated cardiovascular risk factors. ${ }^{28-30}$ The cumulative effects of these disadvantages among women throughout the trajectory of disease could explain some of the excess relative risk.

\section{Can women "catch up" with men?}

An interesting question is under what conditions would rates of MI in men and women be the same? The answer depends on many factors, including the prevalence of the risk factors for MI (see supplementary appendix). In the UK Biobank the rates of MI per 10000 person years in women with hypertension (AHA stage 1 or 2) and diabetes (type 1 or 2) who were also current smokers was 41.76 (95\% confidence interval 25.58 to 68.12) compared with 53.68 (39.95 to 72.13) in men. So even in this extreme group, defined only by risk factors for which relative risks in women exceed those 
in men, in our study population men still have higher rates of MI. Furthermore, few women $(0.2 \%)$ and men $(0.6 \%)$ had this combination of risk factors and their contribution to the overall rate of $\mathrm{MI}$ is inevitably small. It would take an enormous increase in the prevalence of all three risk factors for this subgroup to have even a moderate impact on the overall risk of MI in the future.

\section{Conclusions and policy implications}

Although the risk of MI is, on average, about three times higher in men than women, women tend to "catch up" to some extent if they have certain cardiovascular risk factors. Our findings suggest that clinicians should be vigilant when their female patients are elderly, smoke, have diabetes, or have high blood pressure. These findings also highlight the importance of equitable access to guideline based treatments for diabetes and hypertension, and to weight loss and smoking cessation programmes for women and men in middle and older age.

Despite the rate of MI being higher in men than women, hypertension, smoking (especially higher intensity), and type 1 and 2 diabetes confer a greater excess risk of MI in women than in men. This excess risk does not attenuate with age. In addition, a rising prevalence of lifestyle associated risk factors, coupled with the ageing population, is likely to result in women having a more similar overall rate of MI to men in the future, with a major additional burden on society and health resources.

This research has been conducted using the UK Biobank Resource (application No 2495). Permission to use the UK Biobank Resource was approved by the access subcommittee of the UK Biobank Board.

Contributors: All authors were involved in the design of the study. ERCM carried out the statistical analyses and wrote the first draft of the paper, with support from SAEP and MW. All authors contributed to further drafts and approved the final manuscript. The corresponding author attests that all listed authors meet authorship criteria and that no others meeting the criteria have been omitted. ERCM is the guarantor.

Funding: SAEP is supported by a UK Medical Research Council skills development fellowship (MR/P014550/1). MW is supported by a National Health and Medical Research Council fellowship (APP108026). The funding sources had no role in the design or conduct of the study; collection, management, analysis, and interpretation of the data; or preparation, review, or approval of the manuscript.

Competing interests: All authors have completed the ICMJE uniform disclosure form at www.icmje.org/coi_disclosure.pdf and declare: no support from any organisation for the submitted work; MW does consultancy for Amgen outside the submitted work; no other relationships or activities that could appear to have influenced the submitted work.

Ethical approval: UK Biobank has obtained Research Tissue Bank approval from its governing research ethics committee, as recommended by the National Research Ethics Service. No separate ethical approval was required. The study was conducted in accordance with the principles of the Declaration of Helsinki.

Data sharing: Researchers can apply to use the UK Biobank resource and access the data used. No additional data are available.

Transparency: The manuscript's guarantor (ERCM) affirms that this manuscript is an honest, accurate, and transparent account of the study being reported; that no important aspects of the study have been omitted; and that any discrepancies from the study as planned (and, if relevant, registered) have been explained.

This is an Open Access article distributed in accordance with the terms of the Creative Commons Attribution (CC BY 4.0) license, which permits others to distribute, remix, adapt and build upon this work, for commercial use, provided the original work is properly cited. See: http://creativecommons.org/licenses/by/4.0/.

1 World Health Organization. Top 10 causes of death worldwide Geneva: WHO; 2017. www.who.int/mediacentre/factsheets/fs310/ en/.

2 Institute for Health Metrics and Evaluation. GBD Compare Data Visualization Seattle, WA: IHME, University of Washington; 2016. https://vizhub.healthdata.org/gbd-compare/.

3 Naghavi M, Abajobir AA, Abbafati C, et al, GBD 2016 Causes of Death Collaborators. Global, regional, and national age-sex specific mortality for 264 causes of death, 1980-2016: a systematic analysis for the Global Burden of Disease Study 2016. Lancet 2017;390:1151-210 doi:10.1016/S0140-6736(17)32152-9

4 Bots SH, Peters SAE, Woodward M. Sex differences in coronary heart disease and stroke mortality: a global assessment of the effect of ageing between 1980 and 2010. BMJ Global Health 2017:2:e000298. doi:10.1136/bmjgh-2017-000298

5 Anand SS, Islam S, Rosengren A, et al, INTERHEART Investigators. Risk factors for myocardial infarction in women and men: insights from the INTERHEART study. Eur Heart / 2008;29:932-40. doi:10.1093/eurheartj/ehn018

6 Peters SAE, Huxley RR, Woodward M. Diabetes as risk factor for incident coronary heart disease in women compared with men: a systematic review and meta-analysis of 64 cohorts including 858,507 individuals and 28,203 coronary events. Diabetologia 2014;57:1542-51. doi:10.1007/s00125-014-3260-6

7 Huxley RR, Woodward M. Cigarette smoking as a risk factor for coronary heart disease in women compared with men: a systematic review and meta-analysis of prospective cohort studies. Lancet 2011;378:1297-305. doi:10.1016/S0140-6736(11)60781-2

8 Sudlow C, Gallacher J, Allen N, et al. UK biobank: an open access resource for identifying the causes of a wide range of complex diseases of middle and old age. PLoS Med 2015;12:e1001779. doi:10.1371/journal.pmed.1001779

9 Whelton PK, Carey RM, Aronow WS, et al. ACC/AHA/AAPA/ABC/ ACPM/AGS/APhA/ASH/ASPC/NMA/PCNA Guideline for the Prevention, Detection, Evaluation, and Management of High Blood Pressure in Adults. A Report of the American College of Cardiology/American Heart Association Task Force on Clinical Practice Guidelines. Hypertension 2017;71:e13-e115, doi/10.1161/HYP.0000000000000065.

10 Schnier C, Sudlow C. Definitions of acute myocardial infarction (MI) and main MI pathological types for UK Biobank phase 1 outcomes adjudication 2017 [14 September 2018]. https://biobank.ctsu.ox.ac. uk/crystal/docs/alg_outcome_mi.pdf.

11 Woodward M. Epidemiology: Study Design and Data Analysis. 3rd ed. Chapman and Hall/CRC, 2013 doi:10.1201/b16343

12 Yusuf S, Hawken S, Ôunpuu S, et al, INTERHEART Study Investigators. Effect of potentially modifiable risk factors associated with myocardial infarction in 52 countries (the INTERHEART study): case-control study. Lancet 2004;364:937-52. doi:10.1016/S0140-6736(04)17018-9

13 Peters SAE, Huxley RR, Woodward M. Comparison of the sex-specific associations between systolic blood pressure and the risk of cardiovascular disease: a systematic review and meta-analysis of 124 cohort studies, including 1.2 million individuals. Stroke 2013;44:2394-401. doi:10.1161/STROKEAHA.113.001624

14 Zhou B, Bentham J, Di Cesare M, et al.Worldwide trends in blood pressure from 1975 to 2015: a pooled analysis of 1479 population-based measurement studies with 19.1 million participants. Lancet 2017;389:37-55.

15 Rapsomaniki E, Timmis A, George J, et al. Blood pressure and incidence of twelve cardiovascular diseases: lifetime risks, healthy life-years lost, and age-specific associations in 1.25 million people. Lancet 2014;383:1899-911. doi:10.1016/S0140-6736(14)60685-1

16 Tamargo J, Rosano G, Walther T, et al. Gender differences in the effects of cardiovascular drugs. Eur Heart J Cardiovasc Pharmacother 2017;3:163-82. doi:10.1093/ehjcvp/pvw042

17 Teo KK, Ounpuu S, Hawken S, et al, INTERHEART Study Investigators. Tobacco use and risk of myocardial infarction in 52 countries in the INTERHEART study: a case-control study. Lancet 2006;368:647-58. doi:10.1016/S0140-6736(06)69249-0

18 Iversen B, Jacobsen BK, Løchen M-L. Active and passive smoking and the risk of myocardial infarction in 24,968 men and women during 11 year of follow-up: the Troms $\varnothing$ Study. Eur J Epidemiol 2013;28:659-67. doi:10.1007/s10654-013-9785-z

$19 \mathrm{Ng} \mathrm{M}$, Freeman MK, Fleming TD, et al. Smoking prevalence and cigarette consumption in 187 countries, 1980-2012. IAMA 2014;311:183-92. doi:10.1001/jama.2013.284692 
20 Hitchman SC, Fong GT. Gender empowerment and female-to-male smoking prevalence ratios. Bull World Health Organ 2011;89:195202. doi:10.2471/BLT.10.079905

21 Peters SAE, Huxley RR, Woodward M. Do smoking habits differ between women and men in contemporary Western populations? Evidence from half a million people in the UK Biobank study. BMJ Open 2014;4:e005663. doi:10.1136/bmjopen-2014-005663

22 Ballotari P, Venturelli F, Greci M, Giorgi Rossi P, Manicardi V. Sex Differences in the Effect of Type 2 Diabetes on Major Cardiovascular Diseases: Results from a Population-Based Study in Italy. Int I Endocrinol 2017;2017:6039356. doi:10.1155/2017/6039356

23 Gnatiuc L, Herrington WG, Halsey I, et al, Prospective Studies Collaboration and Asia Pacific Cohort Studies Collaboration. Sex-specific relevance of diabetes to occlusive vascular and other mortality: a collaborative meta-analysis of individual data from 980793 adults from 68 prospective studies. Lancet Diabetes Endocrinol 2018;6:538-46. doi:10.1016/S2213-8587(18)30079-2

24 Bragg F, Holmes MV, Iona A, et al, China Kadoorie Biobank Collaborative Group. Association between diabetes and cause-specific mortality in rural and urban areas of china. JAMA 2017;317:280-9. doi:10.1001/jama.2016.19720

25 Shah AD, Langenberg C, Rapsomaniki E, et al. Type 2 diabetes and incidence of cardiovascular diseases: a cohort study in 1.9 million people. Lancet Diabetes Endocrinol 2015;3:105-13. doi:10.1016/S2213-8587(14)70219-0
26 Roche MM, Wang PP. Sex differences in all-cause and cardiovascular mortality, hospitalization for individuals with and without diabetes, and patients with diabetes diagnosed early and late. Diabetes Care 2013;36:2582-90. doi:10.2337/dc12-1272

27 Wannamethee SG, Papacosta O, Lawlor DA, et al. Do women exhibit greater differences in established and novel risk factors between diabetes and non-diabetes than men? The British Regional Heart Study and British Women's Heart Health Study. Diabetologia 2012;55:80-7. doi:10.1007/s00125-011-2284-4

28 Health and Social Care Information Centre. National Diabetes Audit - 2012-2013: Report 1. Care Processes and Treatment Targets, 2014.

29 Franzini L, Ardigò D, Cavalot F, et al, IT Study Group of the Italian Society of Diabetology. Women show worse control of type 2 diabetes and cardiovascular disease risk factors than men: results from the MIND. Nutr Metab Cardiovasc Dis 2013;23:235-41. doi:10.1016/j.numecd.2011.12.003

30 Winston GJ, Barr RG, Carrasquillo O, Bertoni AG, Shea S. Sex and racial/ethnic differences in cardiovascular disease risk factor treatment and control among individuals with diabetes in the Multi-Ethnic Study of Atherosclerosis (MESA). Diabetes Care 2009:32:1467-9. doi:10.2337/dc09-0260

Supplementary information: supplementary tables $1-9$, and equations 